\title{
Stellate ganglion block improves the proliferation and function of splenic CD4+ T cells through inhibition of post- hemorrhagic shock mesenteric lymph-mediated autophagy
}

\author{
Ying Li \\ Hebei North University \\ Hui-Bo Du \\ Hebei North University \\ Li-Na Jiang \\ Hebei North University \\ Chen Wang \\ Hebei North University \\ Meng Yin \\ Hebei North University \\ Li-Min Zhang \\ Hebei North University
}

Hong Zhang

Hebei North University

Zhen-Ao Zhao

Hebei North University

Zhan-Kuang Liu

Hebei North University First Affiliated Hospital

Chun-Yu Niu ( $\nabla$ ncylxf@126.com )

Hebei North University

Zigang Zhao ( $\nabla$ zzghyl@126.com)

Hebei North University https://orcid.org/0000-0002-9434-5231

\section{Research Article}

Keywords: Hemorrhagic shock, Stellate ganglion block, CD4+ T cells, Post-hemorrhagic shock mesenteric lymph, Autophagy, Spleen

Posted Date: July 1st, 2021 
DOl: https://doi.org/10.21203/rs.3.rs-294651/v1

License: (c) (1) This work is licensed under a Creative Commons Attribution 4.0 International License. Read Full License 


\section{Abstract}

Background and objective

Severe hemorrhagic shock leads to excessive inflammation and immune dysfunction, which resulted in high mortality related to mesenteric lymph return. Recent study showed that stellate ganglion block (SGB) increased survival rate in rats suffered hemorrhagic shock. However, whether SGB ameliorates immune dysfunction induced by hemorrhagic shock, it remains unknown. The aim of present study is therefore to verify the favorable effect of SGB on the proliferation and function of splenic CD4 + T cells isolated from rats underwent hemorrhagic shock, and investigate its mechanism focusing autophagy and PHSML.

Materials and methods

Male rats underwent SGB or sham SGB pretreatment and conscious acute hemorrhage followed by resuscitation and multiple treatments. After three hours of resuscitation, splenic CD $4+T$ cells were isolated for the measurements of proliferation and cytokine production following stimulation with ConA in vitro. Furthermore, the $\mathrm{CD} 4+\mathrm{T}$ cells isolated from normal rats were treated with post-hemorrhagic shock mesenteric lymph (PHSML) drained from rats treated with SGB or not. And the proliferation, cytokine production and autophagy biomarkers were detected.

Results

Hemorrhagic shock reduced CD $4+T$ cells proliferation and function to produce interleukin (IL)-2, IL-4 and tumor necrosis factor-a-induced protein 8 like 2 (TIPE2). SGB pretreatment or administration of autophagy inhibitor 3-methyladenine (3-MA) significantly normalized these indicators. In contrast, administration of autophagy agonist rapamycin (RAPA) or intravenous injection of PHSML inhibited the beneficial effect of SGB on CD4 + T cells obtained from hemorrhagic shocked rats. Furthermore, PHSML incubation decreased the proliferation and cytokine production, increased LC3 II/I and Beclin-1 expressions, and reduced p-PI3K and p-Akt expressions of normal CD4 + T cells. More critically, these adverse effects of PHSML were abolished by 3-MA administration, as well as incubation with PHSML obtained from SGB treated rats.

Conclusions

SGB improves splenic CD4 + T cells function following hemorrhagic shock, which is related to the inhibition of PHSML-mediated autophagy.

\section{Introduction}

Hemorrhagic shock is an acute and critical pathological process, which is more common in traumatic hemorrhage, postpartum hemorrhage, visceral rupture, peptic ulcer, and so on ${ }^{[1]}$. The immune system plays a very important role in maintaining the homeostasis of internal environment. Severe hemorrhagic shock induces hyper-inflammation and immunosuppression, which is a key link in the development of 
hemorrhagic shock leading to sepsis and multiple organ dysfunction ${ }^{[2-5]}$. T cells have always been one of the important regulatory components in the cellular immune response ${ }^{[6,7]}$. Studies have shown that the function of $T$ cells is significantly inhibited after hemorrhagic shock. CD4 + T cell dysfunction has a vital role in the pathogenesis of immunosuppression after hemorrhagic shock. Meanwhile, the role of various inflammatory mediators entering the systemic circulation through mesenteric lymph pathway and causing organ damage has been widely concerned. Blockage of post-hemorrhagic shock mesenteric lymph (PHSML) return reduces splenic histological injury and normalizes cellular immune function ${ }^{8,9]}$. So, PHSML return is a key reason that aggravates multiple organ injury and immune dysfunction $[10,11]$.

Stellate ganglion block (SGB), a common method of blocking sympathetic nerves, plays the effect of reducing the release of inflammatory factors, enhancing the activity of $T$ cells and increasing the secretion of anti-inflammatory cytokines ${ }^{[12]}$. Previous studies have shown that SGB can prolong the survival time of hemorrhagic shock rats and reduce the damage of intestinal barrier function [13]. However, it is unclear that whether SGB affects the function of CD $4+T$ cells after acute hemorrhage. The bidirectional effect of autophagy on cell structure and function has received attention, and excessive autophagy inhibits the differentiation and function of $\mathrm{CD} 4+\mathrm{T}$ cells ${ }^{[14]}$. However, following hemorrhagic shock, whether autophagy occurs in CD $4+T$ cells and relates to PHSML return-induced CD4 $+T$ cells dysfunction, and the relationship between CD4 + T cell autophagy and SGB, it remains unknown. Therefore, we hypothesized that SGB improves splenic CD4 + T cells function through inhibition of PHSML return-mediated autophagy. To test the hypothesis, this study observed the effect of autophagy activator and intravenous PHSML on the effect of SGB on the proliferation and cytokine production of CD4 + T cells, and investigated the effect of PHSML from SGB-treated rat (PHSML-SGB) on above indices and autophagy in CD4 $+\mathrm{T}$ cells isolated from normal rat spleen.

\section{Methods}

\section{Animals}

Male Wistar rats (280-320 g) were purchased from Sibeifu Biotechnology Co., Ltd (Beijing, China), and maintained in a clean-grade animal house. All surgeries were performed under anesthesia according to the Use of Laboratory Animals Guidelines from the National Institutes of Health. The Animal Ethics Committee in Hebei North University approved the experimental procedures.

\section{Experimental procedures}

Firstly, twenty rats underwent pretreatment of SGB and sham SGB $(n=10)$, and then the model of hemorrhagic shock under anesthesia were established for PHSML drainage, the PHSML and PHSML-SGB were stored at $-80^{\circ} \mathrm{C}$ for subsequent experimental research. Secondly, forty-two rats were divided into seven groups ( $n=6$ each group). As follows: Sham group, Shock group, Sham + SGB group, Shock + SGB group, Shock + 3-methyladenine (3-MA) group, Shock + SGB + Rapamycin (RAPA) group, Shock + SGB + PHSML group. The SGB pretreatment and hemorrhagic shock model in conscious rat were performed for 
the isolation and proliferation and cytokine production observation of splenic CD4 + T cells. Thirdly, fifteen rats were used for the isolation of splenic CD4 + T cells. Then, the CD4 + T cells were treated with various mesenteric lymph, including PHSML (4\%), PHSML-SGB (4\%), PHSML (4\%) plus 3-MA (5 mM), and phosphate buffer saline (PBS) or dimethyl sulfoxide (DMSO, 1\%o) as control. The proliferation, cytokine production and autophagy of splenic CD $4+\mathrm{T}$ cells were detected.

\section{SGB pretreatment}

SGB pretreatment was performed in accordance with the method in our laboratory ${ }^{[13]}$. Briefly, each rat was induced inhalation anesthesia with isoflurane, and $0.2 \mathrm{ml}$ ropivacaine hydrochloride (AstraZeneca $A B$, Sweden) of $2.5 \%$ concentration were injected to the body surface landmarks of right stellate ganglion. After the rats naturally awake, the positive Horner syndrome (right side droopy eyelid) was observed for confirmation of SGB. If there was no obvious droopy eyelid, the SGB treatment is considered a failure and the rat was relinquished. The sham SGB treatment was performed with the injection of an equal amount of $0.9 \%$ saline.

\section{Collection of PHSML}

Rats treated with SGB or sham SGB were subjected to hemorrhagic shock model under anesthesia. After induction inhalation anesthesia with isoflurane, the rats were anesthetized with $1 \%$ pentobarbital sodium $(5 \mathrm{~mL} / \mathrm{kg})$, and polyethylene tubing was aseptically cannulated to both bilateral femoral arteries and right femoral vein. One side of the femoral artery is connected to PowerLab biological signal acquisition system (ADInstruments, Bella Vista NSW, Australia) for mean artery pressure (MAP) dynamic monitoring, and the other side is connected to NE-1000 automatic withdrawal-infusion pump (New Era Pump Systems Inc., Farmingdale, NY) for acute hemorrhage. The other pump was connected to the right femoral vein for fluid resuscitation. An incision of about $3 \mathrm{~cm}$ in the middle of the abdomen was made using an electrotome for the mesenteric lymph drainage. After surgery and 30-min stabilization, blood was discharged through the femoral artery until the MAP was $40 \mathrm{mmHg}$ within 10 minutes, followed by maintain for 60 minutes; then resuscitation were carried out through the femoral vein with shed whole blood and Ringer's solution at 1:1 ratio. After resuscitation, the mesenteric lymphatic duct was exposed, and catheterization was performed to drain the mesenteric lymph for 3 hours. The sample of mesenteric lymph was collected and stored in a refrigerator at $-80^{\circ} \mathrm{C}$ for subsequent experiments.

\section{Conscious rat hemorrhagic shock model}

Rats received isoflurane anesthesia before femoral surgery. Both femoral arteries and the right femoral vein were aseptically cannulated with polyethylene tubing. The tubing was led out from the middle of scapula through a subcutaneous tunnel on the back, and was closed with heparin sodium after fixation. The right femoral artery was connected to the PowerLab biological signal acquisition system to monitor MAP, and the contralateral femoral artery was connected to a withdrawal-infusion pump for blood withdrawal. After the surgery and 20-min stabilization period, acute bleeding was carried out rapidly through the left femoral artery at a rate of $0.6 \mathrm{ml} / \mathrm{min}$, and the MAP reached $40 \mathrm{mmHg}$ within 10 minutes and was maintained at $40 \pm 2 \mathrm{mmHg}$ for 1 hour. Subsequently, fluid resuscitation was performed with the 
shed whole blood and equal Ringer's solution within 30 minutes. At the same time, the administrations of 3-MA (1.92 mL/100 g, M9281, Sigma-Aldrich, USA), RAPA (4 mL/kg, A8167, Apexbio, Texas, USA) and PHSML were performed, respectively. The sham rats underwent the same operation, but neither hemorrhage nor fluid resuscitation.

\section{Splenic tissues Management}

At 3 hours after resuscitation in the shocked rats and the corresponding time point in the sham rats, splenic tissues were aseptically removed from rats. The three splenic samples from each group were collected for $\mathrm{CD} 4^{+} \mathrm{T}$ cells isolation for observation of cell proliferation and produce cytokines ability after stimulation of ConA in vitro. The other splenic samples were further used for other experiments (did not show in this study).

\section{CD4 ${ }^{+} \mathrm{T}$ cells isolation}

The collected spleen was placed into a $5 \mathrm{ml}$ EP tube filled with cold phosphate buffer saline (PBS). Then, the spleen was minced and ground with a syringe piston in a $70 \mu \mathrm{m}$ sieve to produce a single cell suspension. After centrifugation at $1500 \mathrm{rpm}$ for 5 minutes, the isolated splenocytes were resuspended with PBS. The splenocytes suspension was carefully added to the upper layer of an equal amount of lymphocyte separation solution with a pipette, and the mixed solution was centrifuged at $3000 \mathrm{rpm}$ for 20 minutes. Then, the cloudiness ring-shaped lymphocyte in the middle layer was carefully pipetted into a new $15 \mathrm{ml}$ centrifuge tube, and wash three times with PBS. After cell counting, resuspended each $10^{7}$ cells with $80 \mu \mathrm{l}$ PBS were mixed with $20 \mu \mathrm{l}$ of CD4 MicroBeads (Miltenyi Biotec, Bergisch Gladbach, Germany) for incubation for 15 minutes at $4{ }^{\circ} \mathrm{C}$. The CD4 MicroBeads labeled CD $4+\mathrm{T}$ cells were obtained by a separation column (Miltenyi Biotec) in the magnetic field of a MACS separator. After washing and centrifugation, the final cell concentration was determined to be $2 \times 10^{6}$ cells / $\mathrm{ml}$ and then resuspended in RPMI 1640 containing $10 \%$ heat-inactivated fetal bovine serum and $1 \%$ antibiotic at $37^{\circ} \mathrm{C}$.

\section{Purity identification of splenic $\mathrm{CD4}^{+} \mathrm{T}$ cells}

The isolated cells $\left(5 \times 10^{5}\right)$ were resuspended in $100 \mu \mathrm{L}$ of PBS, then, the $2 \mu \mathrm{L}$ of $\mathrm{CD}_{3}-\mathrm{PE}$ antibody, $2 \mu \mathrm{L}$ of $\mathrm{CD}_{4}$-FITC antibody, and two antibodies were added simultaneously, respectively. Meanwhile, there was no antibody as negative control. After incubation at $4{ }^{\circ} \mathrm{C}$ for 15 minutes, the purity of $\mathrm{CD} 4^{+} \mathrm{T}$ cell was identified using flow cytometry (C6, BD Biosciences Inc, San Jose, CA). The results from flow cytometry analysis showed that there was exceeding $90 \% \mathrm{CD} 4^{+} \mathrm{T}$ cells contained in isolated cells (Fig. 1), which demonstrated that the isolated cells can be used for subsequent experiment.

\section{Cell proliferation analysis and Cytokines determination}

The $\mathrm{CD} 4^{+} \mathrm{T}$ cells isolated from the rats subjected to acute hemorrhage or corresponding treatments were plated into 96-well plates and stimulated with ConA ( $5 \mu \mathrm{g} / \mathrm{ml}$, Sigma) at $37^{\circ} \mathrm{C}, 5 \% \mathrm{CO}_{2}$ for 48 hours, and 
then incubated with CCK-8 of $10 \mu \mathrm{l}$ for 4 hours. The CD4 + T cells harvested from normal rats were firstly stimulated by ConA for 48 hours and further treatments with PHSML, or PHSML-SGB, or PHSML plus 3MA for 6 hours, and then incubated with CCK for 4 hours. The absorbency was measured using a microplate reader type of SpectraMax M3 (Molecular Devices, San Jose, CA) at $450 \mathrm{~nm}$, which reflects the proliferation of CD $4+T$ cells. The levels of interleukin (IL)-2, IL-4 and tumor necrosis factor-a-induced protein 8 like 2 (TIPE2) in culture supernatant was detected using the rat-specific ELISA kit according to the instructions (Wuhan Pure Biology Co., Ltd. HuBei, China).

\section{Western blot analysis}

The splenic $\mathrm{CD}^{+} \mathrm{T}$ cells derived from normal rats were treated with DMSO, PHSML, PHSML-SGB, PHSML + 3-MA for 6 hours. The proteins were extracted with RIPA lysis buffer and centrifugation at $12,000 \mathrm{rmp}$ for 10 minutes at $4^{\circ} \mathrm{C}$. After quantification with the BCA kit purchased from Beijing Puli Gene Technology Co., Ltd (Beijing, China), the proteins were separated on 10\%-15\% SDS-PAGE gels and then transferred to the polyvinylidene fluoride (PVDF) membranes using a Mini Trans-Blot Electrophoretic Transfer Cell (Bio-rad, USA). The membranes were blocked in TBS-Tween (TBS-T) diluted 5\% nonfat milk for 1 hour and then respectively incubated with the primary antibodies at $4{ }^{\circ} \mathrm{C}$ overnight, respectively. All antibodies were purchased form Abcam (Cambridge, UK), as follows LC3 (ab192890, 1:2000), Beclin-1 (ab192890, 1:2000), AKT (ab179463, 1:10000), phosphorylate-AKT (ab81283, 1:10000), PI3K (ab191606, 1:1000) and phosphorylate-PI3K (ab182651, 1:1000). The membranes were washed three times with TBS-T and incubated with the secondary antibodies conjugated to horseradish peroxidase (HRP) for $1 \mathrm{~h}$, Finally, the image was detected using ImageQuant ${ }^{\mathrm{TM}}$ LAS 4000 (General Electric Company, Boston, MA), and the gray scale of the target protein was quantified using Quantum One v4.6.2 software.

Statistical analysis

Data are presented as means \pm standard error (SE). The significance of difference between groups was analyzed with one-way analysis of variance (ANOVA) followed by Student-Newman-Keuls (SNK) test. The level of statistically significant was considered at $P<0.05$.

\section{Results}

Effects of SGB on the proliferation and cytokine production of splenic CD4 + T cells in hemorrhagic shock rats

The CCK-8 assay (Fig. 2A) and ELISA assay (Fig. 2B, 2C, 2D) revealed that there were no significant differences between the Sham and Sham + SGB groups $(P>0.05)$. The proliferation and IL-2, IL-4, TIPE2 in culture supernatants of the Shock group was significantly lower than that in the Sham group $(P<0.05)$. When the rats were treated with SGB or 3-MA administration, hemorrhagic shock-induced decreases in these indices were obviously enhanced $(P<0.05)$. In addition, RAPA administration or PHSML injection obviously inhibited the effect of SGB on these indices of rats underwent hemorrhagic shock $(P<0.05)$.

Effects of PHSML-SGB on the proliferation and cytokine production of splenic CD $4+T$ cells in normal rats 
In the analysis of splenic CD $4+T$ cells isolated from normal rats, PHSML incubation reduced the proliferation and cytokines production of IL-2, IL-4, TIPE2 compared to the Control and DMSO groups ( $P<$ 0.05), which were significantly increased following administrations of PHSML-SGB or PHSML +3-MA ( $P$ $<0.05)$.

Effects of PHSML-SGB on autophagy of splenic CD4 + T cells isolated from normal rats.

Western Blotting was used for the detection of autophagy markers LC3 II / I and Beclin1 expressions of $\mathrm{CD}^{+}{ }^{+} \mathrm{T}$ cells (Fig. 4A, 4B), and revealed that PHSML treatment significantly upregulated the Beclin-1 expression and increased the conversion of LC3 I to LC3 II compared to the Control and DMSO groups ( $P$ $<0.05)$, which was obviously inhibited by 3-MA treatment and reversed by PHSML-SGB treatment $(P<$ $0.05)$.

Effects of PHSML-SGB on the expressions of p-PI3K and p-AKT in splenic CD $4^{+} \mathrm{T}$ cells isolated from normal rats.

To elucidate the mechanism by which SGB inhibits PHSML-mediated autophagy of CD4 + T cells, the present study examined the expressions of key signaling proteins of PI3K/Akt pathway. The results (Fig. 4C, 4D) showed that PHSML treatment significantly reduced the expression of p-PI3K and p-AKT of splenic CD4 ${ }^{+}$T cells from normal rats $(P<0.05)$, which was obviously enhanced by 3-MA treatment $(P<$ 0.05). The expressions of $\mathrm{p}-\mathrm{PI} 3 \mathrm{~K}$ and $\mathrm{p}-\mathrm{AKT}$ in splenic $\mathrm{CD} 4^{+} \mathrm{T}$ cells treated with PHSML-SGB were significantly increased than those in PHSML group $(P<0.05)$.

\section{Discussion}

The current study demonstrated that SGB pretreatment or autophagy inhibitor 3-MA administration inhibited the reduction of proliferation and cytokine secretion ability of splenic CD4 + T cells following hemorrhagic shock, and intravenous of PHSML and autophagy agonist RAPA administration eliminated the beneficial effects of SGB. PHSML decreased the proliferation and cytokine production of CD $4+T$ cells isolated normal rats, increased expression of autophagy marker proteins, and inhibited the phosphorylation of PI3K and AKT proteins, which was inhibited by 3-MA treatment. More importantly, the effect of PHSML-SGB was opposite to that of PHSML, and the indicators of CD4 + T cells were close to normal. The present findings suggest that SGB improves the proliferation and function of $\mathrm{CD} 4^{+} \mathrm{T}$ cells after hemorrhagic shock by reducing PHSML-mediated autophagy.

Previous report revealed that the capability of proliferating and producing effector cytokines of CD4 + T cells are the key to performing a variety of effector functions and immune responses ${ }^{[15]}$. Low proliferative response of $\mathrm{CD} 4+\mathrm{T}$ cells is involved in the depressed immune responses after traumainduced hemorrhagic shock ${ }^{[16]}$. After $T$ cell activation and polarization into various $T$ helper subsets, $\mathrm{CD} 4+\mathrm{T}$ lymphocytes can produce IL-2 and IL-4 ${ }^{[17]}$. In the meantime, TIPE-2 is required for maintaining immune homeostasis ${ }^{[18]}$. In vivo, the hypo-secretion of IL-2, IL-4 and TIPE-2 leads to down-regulation of 
immune function ${ }^{[17]}$. Therefore, in this study, we measured the proliferation and cytokines production function of ConA-stimulated CD4 + T lymphocytes, and found that hemorrhagic shock decreased the proliferation capacity and cytokines IL-2, IL-4 and TIPE-2 levels in the culture supernatant of CD $4^{+} \mathrm{T}$ cells stimulated by ConA in vitro, which may be one of the factors that cause the immune system dysfunction and MODS.

Neuromodulation is extremely important for internal environment disturbances caused by hemorrhagic shock. The hypothalamic-pituitary- adrenal (HPA) axis and hypothalamic-sympathetic-adrenal axis are the two key axes for the neuromodulation, as well as affecting the immune response ${ }^{[19]}$. In recent years, neuro-immune interactions have received increasing attention ${ }^{[20,21]}$, the role of the sympathetic nervous system in the immune response has been gradually elucidated ${ }^{[22]}$. For example, adrenergic agonists modulate immune responses in vitro, including cytokine production, lymphocyte proliferation and antibody secretions ${ }^{[23-25]}$, and chemical sympathectomy changes the immune response ${ }^{[26]}$. Under stress, the body first activates the neuro-endocrine regulatory system ${ }^{[27]}$, which regulates immune and endocrine system functions through the HPA axis and hypothalamus-sympathetic nerve-adrenal axis ${ }^{[28]}$. Studies have shown that SGB changes the distribution of lymphocyte subpopulations by blocking excessive excitability of sympathetic nerves, increases the proportion of CD4 + T cells ${ }^{[29]}$, and inhibits excessive inflammatory responses early ${ }^{[12]}$, thereby regulating the inflammatory response and immune function homeostasis. Therefore, the implementation of SGB in advance is conducive to reducing the excessive excitement of sympathetic nerves caused by hemorrhagic shock. However, whether SGB affect the function of CD4 + T cells following hemorrhagic shock, it is unknown. The current findings demonstrated that SGB significantly ameliorated the proliferation and cytokine production function of splenic CD4 + T cells in hemorrhage shocked rats, and suggested that SGB pretreatment is beneficial for regulating $\mathrm{CD} 4+\mathrm{T}$ cell function.

Studies have shown that PHSML return after hemorrhagic shock is a factor that reduces spleen $\mathrm{CD} 4^{+} \mathrm{T}$ cell function and causes immune dysfunction, reducing PHSML return mitigates spleen histological damage and improve immune function ${ }^{[8,9]}$. In order to determine whether the benefits of SGB are achieved through the intestinal lymphatic pathway, we injected PHSML intravenously into SGB-treated hemorrhagic shock rats, and found that the infusion of PHSML abolished the beneficial effects of SGB, and reduced the proliferation and the cytokines secretion of $\mathrm{CD} 4^{+} \mathrm{T}$ cells. Using splenic $\mathrm{CD} 4^{+} \mathrm{T}$ cells extracted from normal rats, it was found that PHSML treatment reduced the proliferation activity and ability to secrete cytokines of CD4 + T cells. In contrast, PHSML-SGB treated cells have significantly increases in proliferation and cytokines IL-2, IL-4 and TIPE-2. Hence, the beneficial effects of SGB are related to the intestinal lymphatic pathway.

Autophagy is a highly conservative cellular biological process, and is one of the two major degradation systems in eukaryotic cells. It is involved in cell proliferation and physiological apoptosis. Its main role is to protect cells under stress. However, the excessive autophagy leads to cell injury, and involves in the development of sepsis ${ }^{[30]}$ and immune response ${ }^{[31]}$. After hemorrhagic shock, splenocytes damage may 
be associated with excessive cellular autophagy ${ }^{[32]}$. The present animal experiments showed that treatment with autophagy inhibitor 3-MA restored the proliferation and the ability to secrete cytokines of $\mathrm{CD} 4^{+} \mathrm{T}$ cells following hemorrhagic shock. Conversely, the autophagy agonist RAPA offset the beneficial effects of SGB. Therefore, inhibition of excessive autophagy is involved in the role of SGB in improving the function of $\mathrm{CD} 4^{+} \mathrm{T}$ cells.

During formation of autophagy, the double membrane organelles called autophagosomes transfer cytoplasmic material to lysosomes ${ }^{[33]}$. In mammalian cells, the formation of autophagosomes is through the release of ATG6 / Beclin-1 from Bcl-2 to form a Vps34-PI3K complex containing Beclin-1, which is essential for the production of autophagosomes ${ }^{[34]}$. Autophagy can be stimulated by starvation and other stresses, various pathological conditions, or drugs such as rapamycin ${ }^{[35,36]}$. When autophagy is excessively induced, it will cause autophagic cell death, so-called type II apoptosis ${ }^{[37]}$. Therefore, in addition to physiological regulatory functions, autophagy is also closely related to inflammation and the occurrence and development of diseases ${ }^{[38]}$. Normal levels of autophagy can maintain the homeostasis of the body's environment, while excessive autophagy can cause apoptosis ${ }^{[39]}$. During autophagosome formation, as a classic marker of autophagosome, the increase of LC3-II represents the beginning of autophagy ${ }^{[40]}$. At the same time, the expression level of Beclin-1 also reflects the intensity of autophagy and become one of the autophagy marker proteins ${ }^{[41]}$.

In order to further clarify the mechanism of cell autophagy, in this study, splenic CD $4+T$ cells from normal rats were cultured with ConA for 48 hours in vitro and then incubated with PHSML. We found that PHSML increased the protein expression of LC3 II/I and Beclin-1 in CD $4+T$ cells. At the same time, the autophagy inhibitor 3-MA significantly reduced the conversion of LC3 I to LC3 II and the expression of Beclin-1 in CD $4+T$ cells, suggesting that PHSML activated autophagy of CD $4+T$ cells. Furthermore, PHSML-SGB treatment significantly reduced the expressions of autophagy marker proteins, suggesting that SGB reduced the autophagy of CD4 + T cells through the intestinal lymphatic pathway.

As is well known that PI3K/AKT pathway is the classic pathway of autophagy. More and more studies showed that PI3K/Akt signaling pathway is a primary pathway that inhibits autophagy ${ }^{[42,43]}$. This study further confirmed that PHSML inhibits the phosphorylation of PI3K and Akt in CD4 + T cells, which was reversed by the PHSML-SGB and PHSML + 3-MA treatments. These results indicated that the effects of PHSML inducing autophagy and SGB reducing autophagy were related to the PI3K / AKT signaling pathway.

In summary, our experimental data demonstrated that SGB improves the function of $\mathrm{CD} 4^{+} \mathrm{T}$ cells after hemorrhagic shock by targeting the PI3K / AKT pathway and reducing cell autophagy, which has a positive significance for maintaining the immune homeostasis, and the role is related to the intestinal lymphatic pathway. However, it is unclear whether SGB also has a therapeutic effect on hemorrhagic shock through other molecular mechanisms. This study focusing on $\mathrm{CD} 4^{+} \mathrm{T}$ cells, provides a certain 
basis for enriching the pathogenesis of hemorrhagic shock-induced immune dysfunction, and also provides a new therapeutic target for the prevention and treatment of severe hemorrhagic shock.

\section{Declarations}

\section{Ethics approval and consent to participate}

All the protocols were approved by the Animal Care Committee of Hebei North University (Zhangjiakou, China).

\section{Consent for publication}

All the authors agree to publish this manuscript.

\section{Availability of data and materials}

The data generated for this study are available on request to the corresponding author.

\section{Competing interests}

No benefits in any form have been received or will be received from a commercial association related directly or indirectly to the subject of this article. The authors report no conflicts of interest. The authors alone are responsible for the content and writing of the paper.

\section{Funding}

This study was supported by the National Natural Science Foundation of China (No. 81770492).

\section{Authors' contributions}

Y.L., H.D., L.J., C.W., M.Y., L.Z., H.Z., Z.Z., and Z.L., performed majority of the animal experiment and laboratory work; Y.L., acquired and analyzed the data; C.N., and Z.Z., were involved in the conception and design of the study, data interpretation, and critically revised the manuscript.

\section{Acknowledgements}

Not Applicable.

\section{References}

1. Cannon, J. W. 2018. Hemorrhagic Shock[J]. N Engl J Med 378 (4): 370-379.

2. Brochner, A. C., and P. Toft. 2009. Pathophysiology of the systemic inflammatory response after major accidental trauma[J]. Scand J Trauma Resusc Emerg Med 17: 43. 
3. Menger, M. D., and B. Vollmar. 2004. Surgical trauma: hyperinflammation versus immunosuppression?[J]. Langenbecks Arch Surg 389 (6): 475-484.

4. Moore, F. A., B. A. McKinley, and E. E. Moore. 2004. The next generation in shock resuscitation[J]. Lancet 363 (9425): 1988-1996.

5. Yao, F., Y. Q. Lu, and J. K. Jiang, et al. 2017. Immune recovery after fluid resuscitation in rats with severe hemorrhagic shock[J]. J Zhejiang Univ Sci B 18 (5): 402-409.

6. de St Groth, B. F. 2012. Regulatory T-cell abnormalities and the global epidemic of immunoinflammatory disease[J].. Immunol Cell Biol 90 (3): 256-259.

7. Katlama, C., S. G. Deeks, and B. Autran, et al. 2013. Barriers to a cure for HIV: new ways to target and eradicate HIV-1 reservoirs[J]. Lancet 381 (9883): 2109-2117.

8. Liu, H., Z. G. Zhao, and L. Q. Xing, et al. 2015. Post-shock mesenteric lymph drainage ameliorates cellular immune function in rats following hemorrhagic shock[J]. Inflammation 38 (2): 584-594.

9. Tiesi, G., D. Reino, and L. Mason, et al. 2013. Early trauma-hemorrhage-induced splenic and thymic apoptosis is gut-mediated and toll-like receptor 4-dependent[J]. Shock (Augusta, Ga.) 39 (6): 507513.

10. Deitch, E. A. 2010. Gut lymph and lymphatics: a source of factors leading to organ injury and dysfunction[J]. Ann N Y Acad Sci 1207 (Suppl 1): E103-E111.

11. Deitch, E. A. 2012. Gut-origin sepsis: evolution of a concept[J]. The surgeon : journal of the Royal Colleges of Surgeons of Edinburgh and Ireland 10 (6): 350-356.

12. Yang, X., Z. Shi, and X. Li, et al. 2015. Impacts of stellate ganglion block on plasma NF-kappaB and inflammatory factors of TBI patients[J]. Int J Clin Exp Med 8 (9): 15630-15638.

13. Zhang, J., X. R. Lin, and Y. P. Zhang, et al. 2019. Blockade of stellate ganglion remediates hemorrhagic shock-induced intestinal barrier dysfunction[J]. J Surg Res 244: 69-76.

14. Jacquin, E., and L. Apetoh. 2018. Cell-Intrinsic Roles for Autophagy in Modulating CD4 T Cell Functions[J]. Front Immunol 9: 1023.

15. Cronkite, D. A., and T. M. Strutt. The Regulation of Inflammation by Innate and Adaptive Lymphocytes[J]. J Immunol Res, 2018, 2018: 1467538.

16. Angele, M. K., and I. H. Chaudry. 2005. Surgical trauma and immunosuppression: pathophysiology and potential immunomodulatory approaches[J]. Langenbecks Arch Surg 390 (4): 333-341.

17. Bagley, J., T. Sawada, and Y. Wu, et al. 2000. A critical role for interleukin 4 in activating alloreactive CD4 T cells[J]. Nat Immunol 1 (3): 257-261.

18. Sun, H., S. Gong, and R. J. Carmody, et al. 2008. TIPE2, a negative regulator of innate and adaptive immunity that maintains immune homeostasis[J]. Cell 133 (3): 415-426.

19. Bellavance, M. A., and S. Rivest. 2014. The HPA - Immune Axis and the Immunomodulatory Actions of Glucocorticoids in the Brain[J]. Front Immunol 5: 136.

20. Vasanthakumar, A., D. Chisanga, and J. Blume, et al. Sex-specific adipose tissue imprinting of regulatory T cells[J]. Nature, 2020. 
21. Kipnis, J. 2016. Multifaceted interactions between adaptive immunity and the central nervous system[J]. Science 353 (6301): 766-771.

22. Mravec, B. 2019. Chemical sympathectomy attenuates lipopolysaccharide-induced increase of plasma cytokine levels in rats pretreated by ACTH[J]. J Neuroimmuno/ 337: 577086.

23. Moriyama, S., J. R. Brestoff, and A. L. Flamar, et al. 2018. beta2-adrenergic receptor-mediated negative regulation of group 2 innate lymphoid cell responses[J]. Science 359 (6379): 1056-1061.

24. Vujnovic, I., I. Pilipovic, and N. Jasnic, et al. 2019. Noradrenaline through beta-adrenoceptor contributes to sexual dimorphism in primary CD4 + T-cell response in DA rat EAE model?[J]. Cell Immuno/ 336: 48-57.

25. Qiao, G., M. J. Bucsek, and N. M. Winder, et al. 2019. beta-Adrenergic signaling blocks murine CD8(+) T-cell metabolic reprogramming during activation: a mechanism for immunosuppression by adrenergic stress[J].. Cancer Immunol Immunother 68 (1): 11-22.

26. Horvathova, L., A. Tillinger, and I. Sivakova, et al. 2015. Chemical sympathectomy increases neutrophil-to-lymphocyte ratio in tumor-bearing rats but does not influence cancer progression[J].. $J$ Neuroimmunol 278: 255-261.

27. Mani, S. K. 2018. Neuroendocrine regulation of reproduction, stress, inflammation and energy homeostasis[J]. J Neuroendocrinol 30 (10): e12648.

28. Smith, S. M., and W. W. Vale. 2006. The role of the hypothalamic-pituitary-adrenal axis in neuroendocrine responses to stress[J].. Dialogues Clin Neurosci 8 (4): 383-395.

29. Yokoyama, M., H. Nakatsuka, and Y. Itano, et al. 2000. Stellate ganglion block modifies the distribution of lymphocyte subsets and natural-killer cell activity[J]. Anesthesiology 92 (1): 109-115.

30. Yin, X., H. Xin, and S. Mao, et al. 2019. The role of autophagy in sepsis: protection and injury to organs[J]. Front Physiol 10: 1071.

31. Tao, S., and I. Drexler. 2020. Targeting autophagy in innate immune cells: angel or demon during infection and vaccination?[J]. Front Immunol 11: 460.

32. Zhang, L., J. S. Cardinal, and P. Pan, et al. 2012. Splenocyte apoptosis and autophagy is mediated by interferon regulatory factor 1 during murine endotoxemia[J]. Shock (Augusta, Ga.) 37 (5): 511-517.

33. Rao, Z., X. Pan, and H. Zhang, et al. 2017. Isoflurane preconditioning alleviated murine liver ischemia and reperfusion injury by restoring AMPK/mTOR-mediated autophagy[J]. Anesth Analg 125 (4): 1355-1363.

34. Yang, Z., and D. J. Klionsky. 2010. Mammalian autophagy: core molecular machinery and signaling regulation[J]. Curr Opin Cell Biol 22 (2): 124-131.

35. Mizushima, N., and M. Komatsu. 2011. Autophagy: renovation of cells and tissues[J].. Cell 147 (4): 728-741.

36. Ravikumar, B., S. Sarkar, and J. E. Davies, et al. 2010. Regulation of mammalian autophagy in physiology and pathophysiology[J]. Physiol Rev 90 (4): 1383-1435. 
37. Chen, Y., and D. J. Klionsky. 2011. The regulation of autophagy - unanswered questions[J].. J Cell Sci 124 (Pt 2): 161-170.

38. Saha, S., D. P. Panigrahi, and S. Patil, et al. 2018. Autophagy in health and disease: A comprehensive review[J]. Biomed Pharmacother 104: 485-495.

39. Chung, Y., J. Lee, and S. Jung, et al. 2018. Dysregulated autophagy contributes to caspase-dependent neuronal apoptosis[J].. Cell Death Dis 9 (12): 1189.

40. Wang, S., X. Xu, and Y. Hu, et al. 2019. Sotetsuflavone Induces Autophagy in Non-Small Cell Lung Cancer Through Blocking PI3K/Akt/mTOR Signaling Pathway in Vivo and in Vitro[J]. Frontiers in Pharmacology 10: 1460.

41. Maejima, Y., M. Isobe, and J. Sadoshima. 2016. Regulation of autophagy by Beclin 1 in the heart[J]. $J$ Mol Cell Cardiol 95: 19-25.

42. Fan, X. J., Y. Wang, and L. Wang, et al. 2016. Salidroside induces apoptosis and autophagy in human colorectal cancer cells through inhibition of PI3K/Akt/mTOR pathway[J]. Oncol Rep 36 (6): 35593567.

43. Wang, Z., L. Zhou, and X. Zheng, et al. 2017. Autophagy protects against PI3K/Akt/mTOR-mediated apoptosis of spinal cord neurons after mechanical injury[J]. Neurosci Lett 656: 158-164.

\section{Figures}
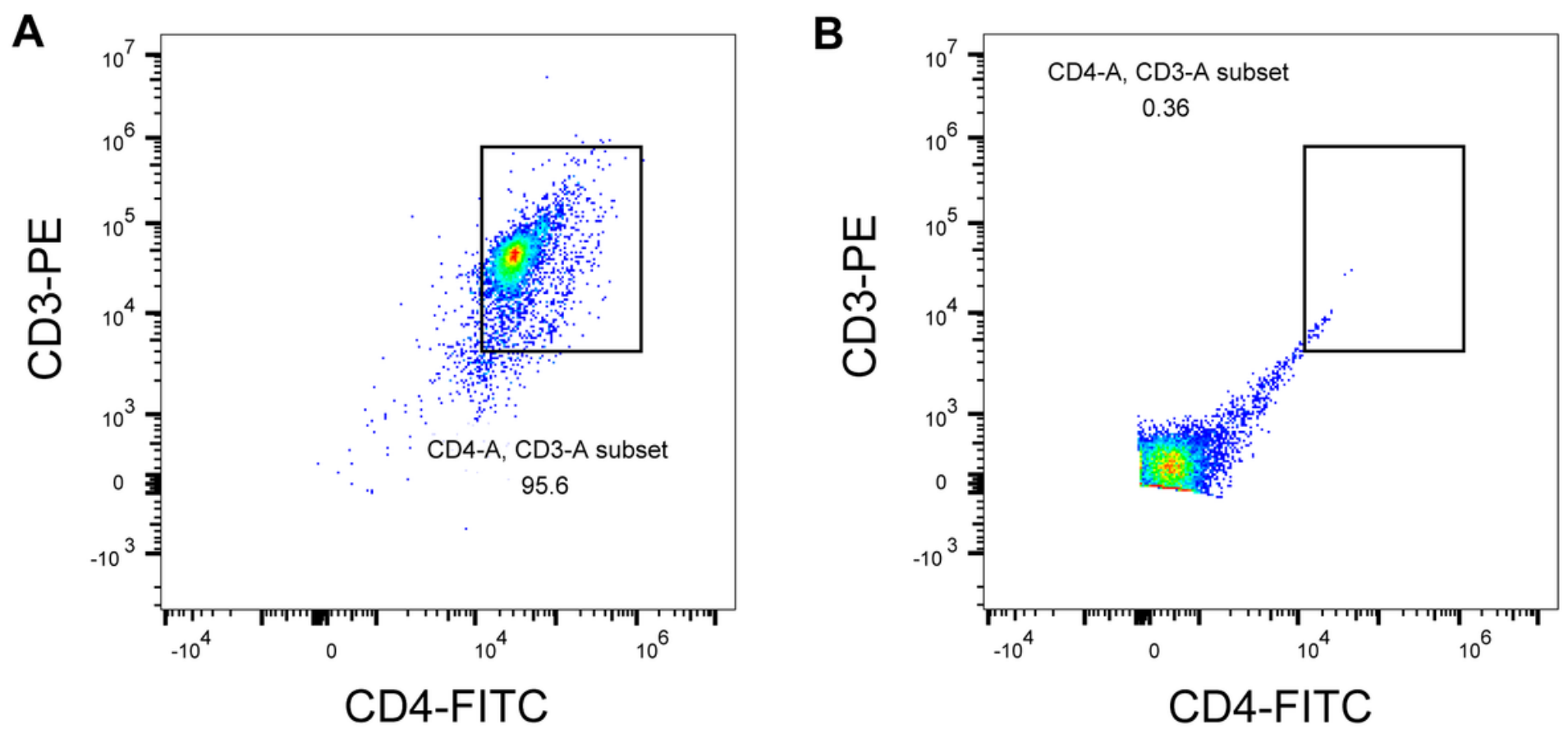

\section{Figure 1}

The purity of splenic CD4+ T cells isolated from the rats. The CD4+ $T$ cells were harvested from spleen in rats with the method of immunomagnetic beads separation techniques, and the purity was indicated with 
flow cytometry analysis. Figure A) showed that the purity of CD4+ T cells was over than $90 \%$. Figure B) is the negative control group.
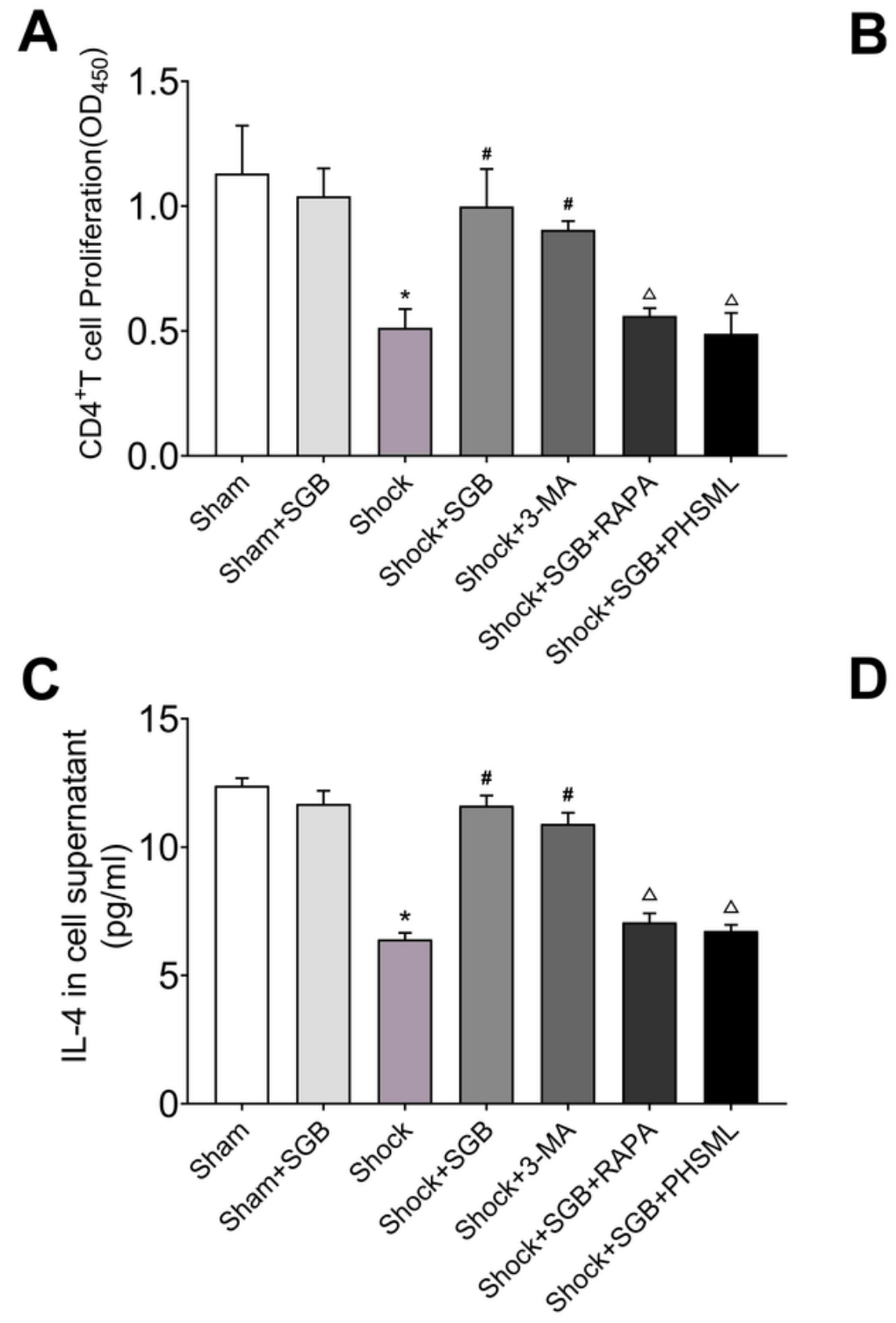

B
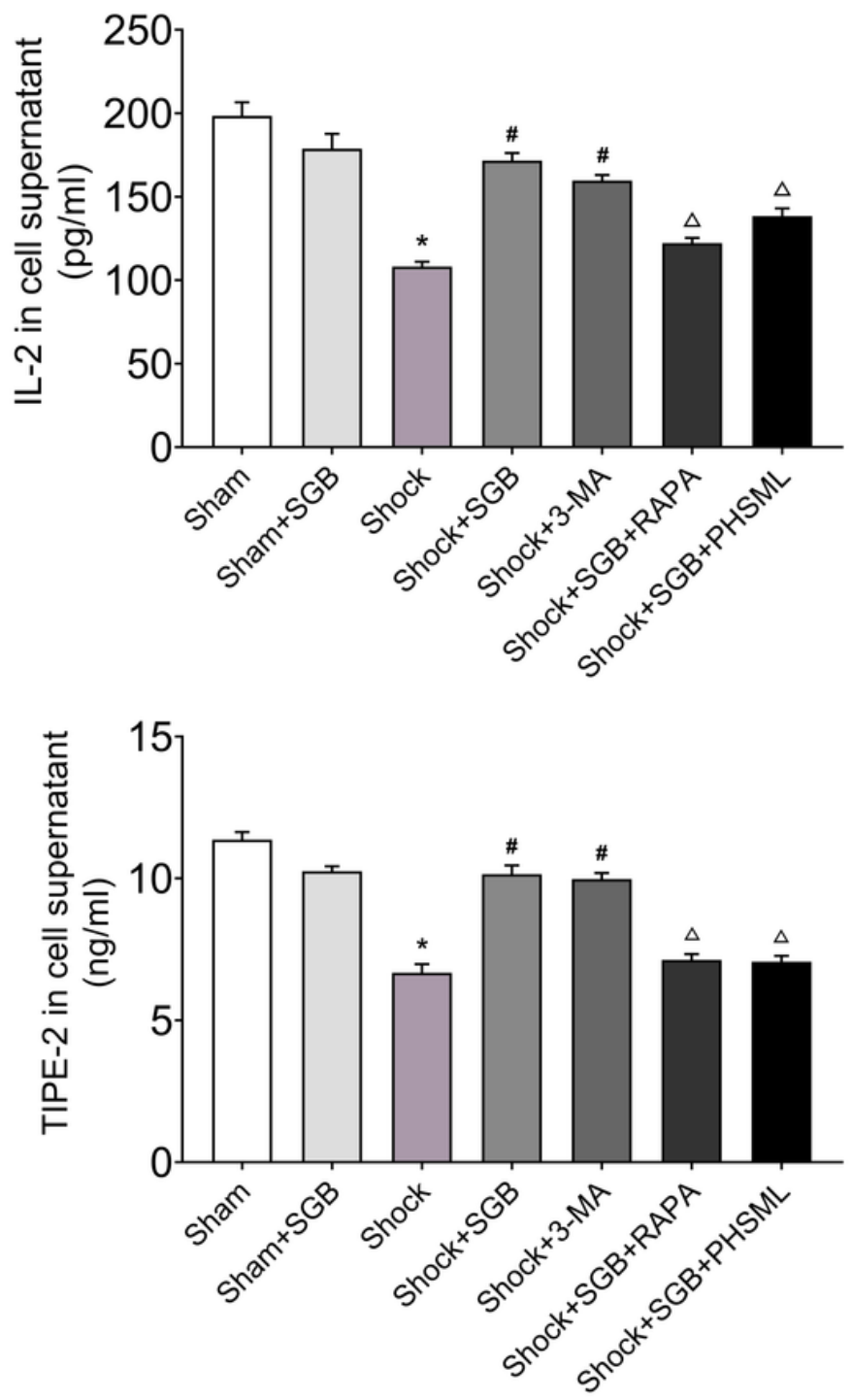

Figure 2

Proliferation and cytokine production of splenic CD4+ T cells isolated from conscious hemorrhagic shock rats. The splenic CD4+ T cells were isolated from conscious hemorrhagic shock rats, and stimulated with ConA $(5 \mu \mathrm{g} / \mathrm{mL})$ for 48 hours in vitro. Figure A) showed the proliferation of splenic CD4+ T cells by the CCK-8 analysis following incubation with CCK-8 for 4 hours. Figure B), C) and D) showed the changes of interleukin (IL)-2, IL-4 and tumor necrosis factor-a-induced protein 8 like 2 (TIPE2) in culture supernatants with the method of ELISA. Data are presented as the mean $\pm S E(n=3) . * P<0.05$ vs Sham group, \# $P<0.05$ vs Shock group, $\triangle P<0.05$ vs Shock+SGB group. 
A
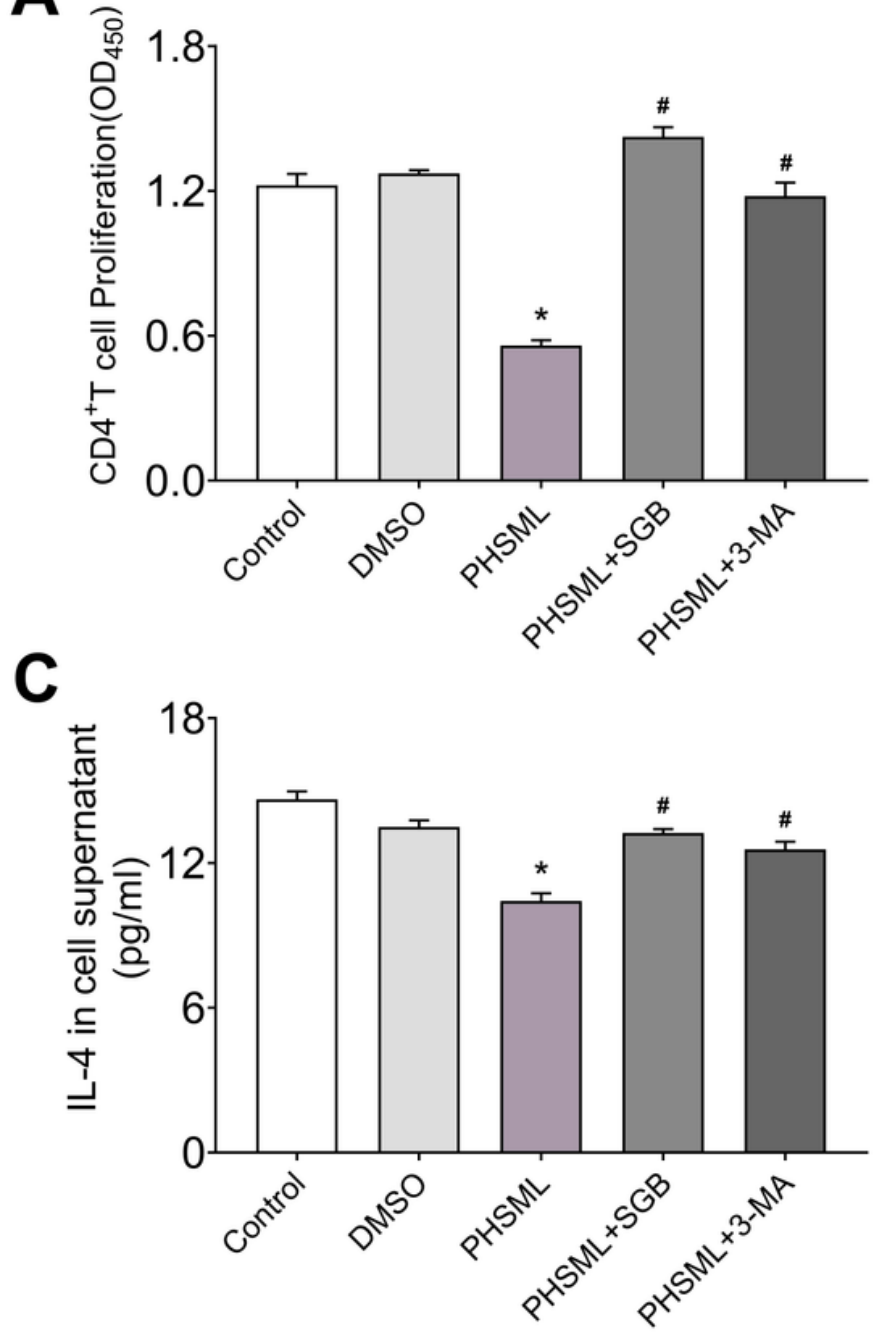

B

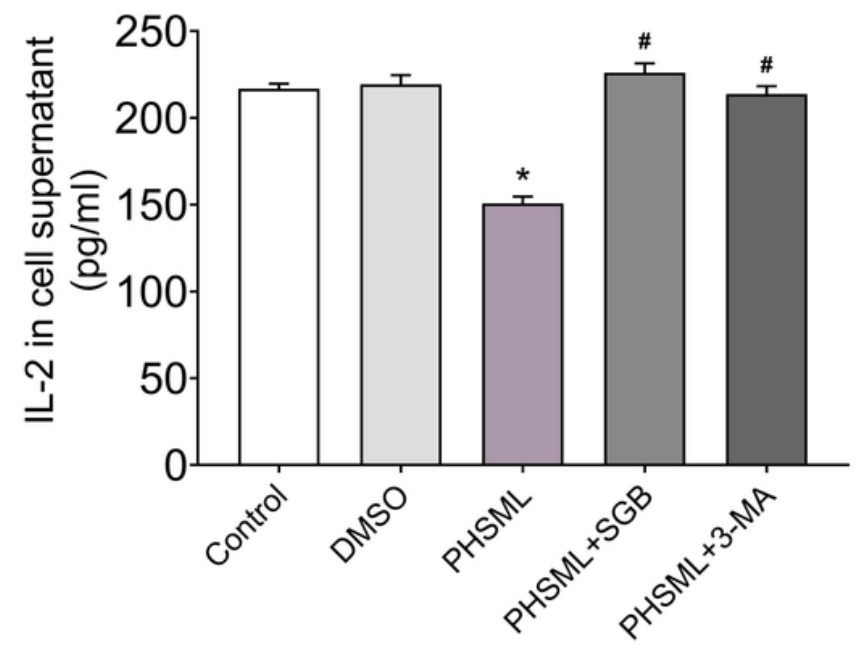

D

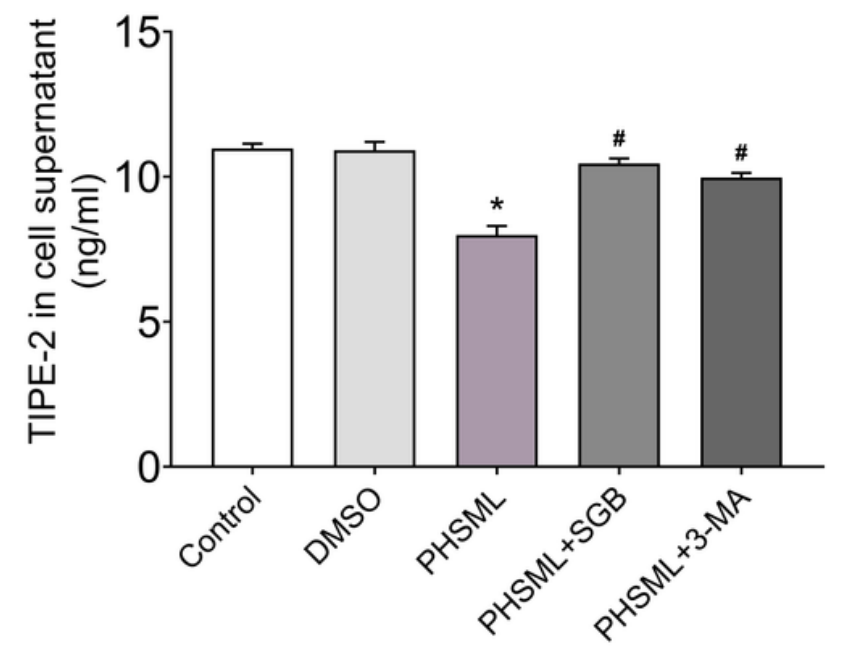

Figure 3

Proliferation and cytokine production of splenic CD4+ T cells isolated from normal rats following stimulation with various post-hemorrhagic shock mesenteric lymph (PHSML). The splenic CD4+ T cells isolated from normal rats were stimulated with ConA $(5 \mu \mathrm{g} / \mathrm{ml})$ for 48 hours and PHSML, or PHSML-SGB, or PHSML plus 3-MA for 6 hours in vitro. Figure A) showed the proliferation of splenic CD4+ T cells by the CCK-8 analysis following incubation with CCK-8 for 4 hours. Figure B), C) and D) showed the changes of interleukin (IL)-2, IL-4 and tumor necrosis factor-a-induced protein 8 like 2 (TIPE2) in culture supernatants with the method of ELISA. Data are presented as the mean $\pm S E(n=3)$. ${ }^{P}<0.05$ vs Control group, \# $P<0.05$ vs PHSML group. 
A
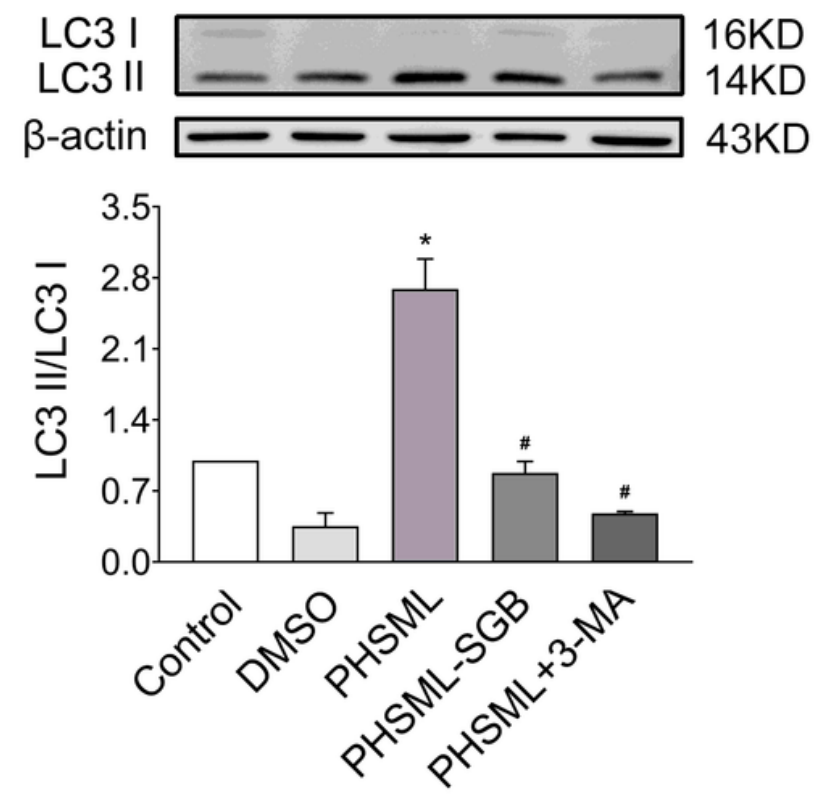

C
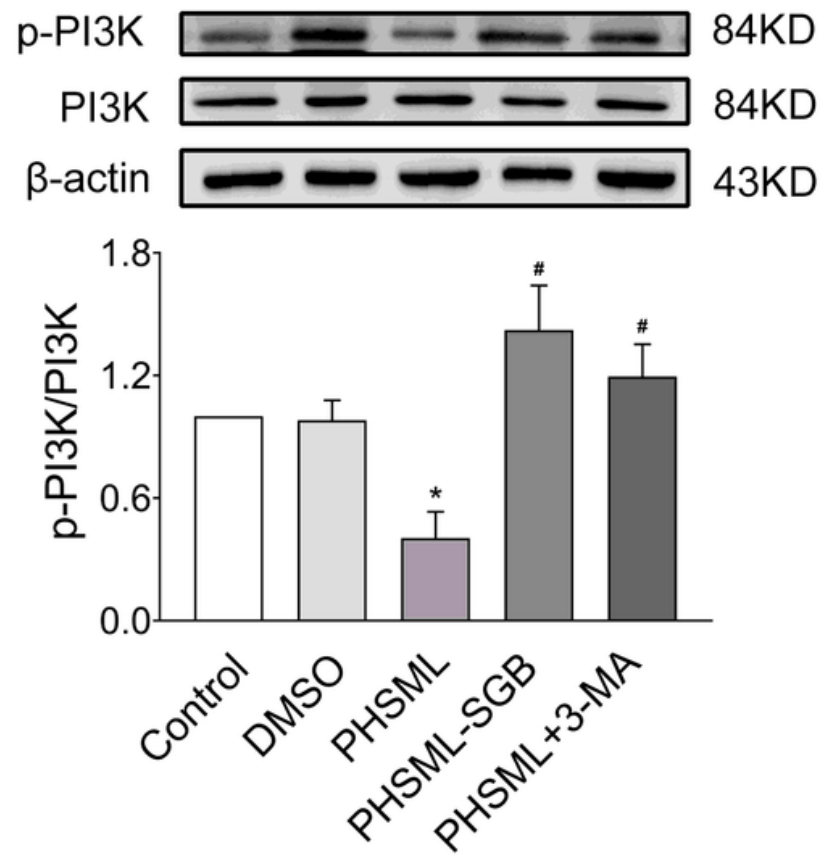

B
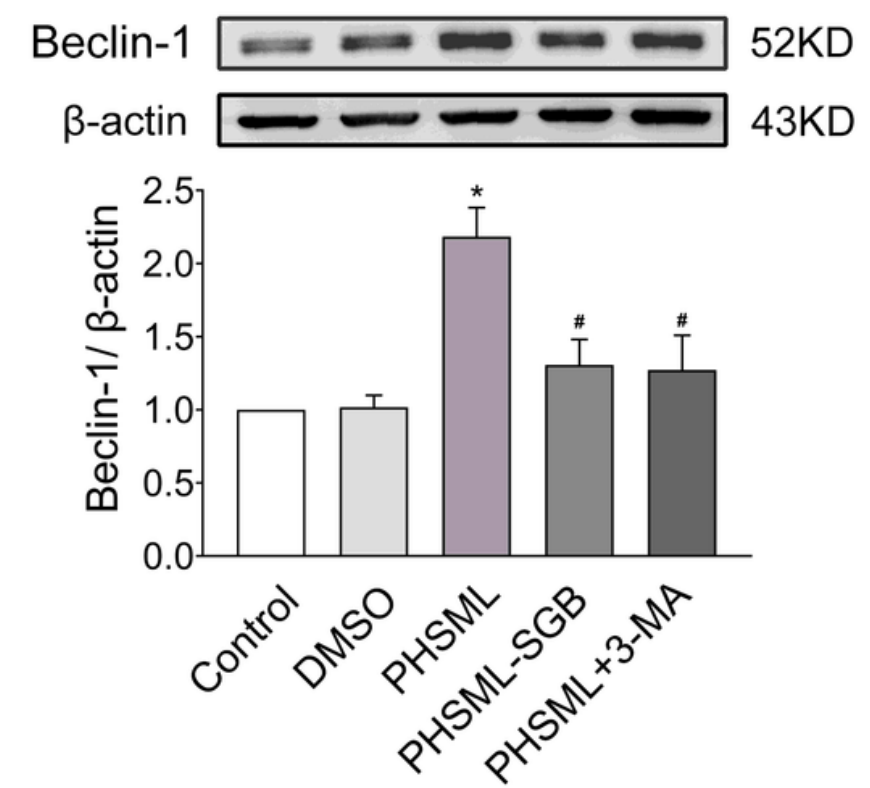

D
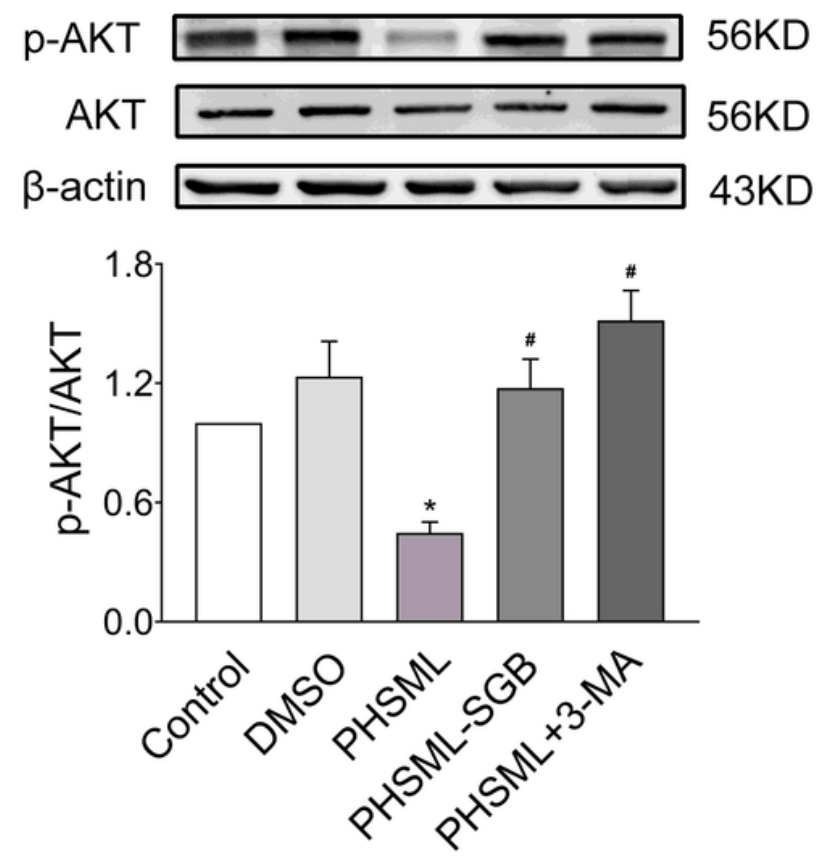

Figure 4

Expressions of LC3 II/I, Beclin-1, p-PI3K, and p-AKT in splenic CD4+ T cells isolated from normal rats following stimulation with various post-hemorrhagic shock mesenteric lymph (PHSML). The splenic CD4+ T cells were isolated from normal rats and stimulated with PHSML, or PHSML-SGB, or PHSML plus 3-MA for 6 hours in vitro. The expressions of autophagy marker protins LC3 II/I and Beclin-1 and the signaling molecules PI3K, p-PI3K, AKT, p-AKT were examined by western blot analysis. Data are presented as the mean $\pm S E(n=3) .{ }^{*}<<0.05$ vs Control group, $\# P<0.05$ vs $P H S M L$ group. 